\title{
Investigation of Novel c-Kit-expressing Smooth Muscle Cells in Murine Cecum
}

\author{
Satoshi Iino, Kazuhide Horiguchi and Satomi Horiguchi \\ Department of Anatomy, University of Fukui Faculty of Medical Sciences, Eiheiji, Fukui 910-1193, Japan
}

Received January 30, 2020; accepted March 3, 2020; published online April 9, 2020

\begin{abstract}
In the gastrointestinal tract musculatures, c-Kit receptor tyrosine kinase is specifically expressed in interstitial cells of Cajal (ICC). ICC are distributed among the smooth muscle cells and are either bipolar or multipolar in shape. Our previous and current study shows that c-Kit-immunopositive smooth muscle cells are present in the murine cecum. Here, we found that c-Kit-expressing smooth muscle cells (named Kit-SM cells) are situated at the submucosal surface of the circular muscle layer. These cells showed smooth muscle actin and myosin immunoreactivities and ultrastructural features such as thick and thin filaments and caveolae. Kit-SM cells also expressed TMEM16A and LRIG1, which are known to be expressed in ICC. Although the functional significance of Kit-SM cells has yet to be revealed, these cells can be considered to have proliferation or differentiation potential in the cecal musculature.
\end{abstract}

Key words: smooth muscle cell, interstitial cells of Cajal, c-Kit, cecum

\section{Introduction}

The proto-oncogene $c$-kit encodes a receptor tyrosine kinase c-Kit and is allelic with white-spotting locus $(W)$ on chromosome 5 in mice. Development of hematopoietic cells, germ cells, melanocytes, mast cells and interstitial cells of Cajal (ICC) depend on signaling from c-Kit and its ligand stem cell factor. In the mammalian gastrointestinal (GI) tract, ICC are divided into several subtypes based on their histological localization and functions [8, 23, 24]; ICC in the circular and longitudinal muscle (ICC-IM) or in the deep muscular plexus layer of the small intestine (ICCDMP) that transduce enteric motor neurotransmission to smooth muscle cells, ICC in the layer of the myenteric plexus (ICC-MY) or on the submucosal surface of the circular muscle (ICC-SM) that show a multipolar shape and serve as pacemakers by generating slow waves and ICC in the subserosal layer of the colon (ICC-SS) that have multipolar shape adjacent to mesothelial cells [27].

Though ICC are different from smooth muscle cells, they regulate the motility of GI tract. To date, ICC have

Correspondence to: Satoshi Iino, Department of Anatomy, University of Fukui Faculty of Medical Sciences, Eiheiji, Fukui 910-1193, Japan.

E-mail: iinosa@u-fukui.ac.jp been determined and observed with c-Kit molecule as antigen or reporter molecule. To investigate ICC, many researchers use c-Kit antibodies or c-Kit reporter mice such as c-Kit-copGFP [22]. Recent studies showed that all ICC in human and mice expressed TMEM16A (Anoctamin-1), which is a transmembrane protein that serves as a calciumactivated chloride channel [4, 6]. TMEM16A is expressed in smooth muscle cells in airway, urogenital and vascular organs, but only in ICC in the GI tract [5].

In the cecum, ICC are located in not only musculature (ICC-IM and ICC-MY), but also in submucosa known as ICC-SP [26]. ICC in submucosal layer are observed in stomach and ileocecal junction in guinea-pig [15, 19]. Our previous study using mice did not reveal ICC in the cecal submucosal layer [9]. On the other hand, we observed cKit-immunopositive cells at the submucosal border of musculature of murine cecum, as observed in colon (ICC on submucosal surface of the circular muscle: ICC-SM) [9]. In this study, we carefully examined the submucosa and the musculature of murine cecum using several methods and identified c-Kit-expressing smooth muscle cells at the submucosal surface of the circular muscle layer. 


\section{Materials and Methods}

\section{Animals}

c-Kit-copGFP mice (Stock No: 015813, B6.129S7$\left.K^{\text {tmlRosay }} / \mathrm{J}\right)$ [22] ( $\mathrm{n}=5$, male) were obtained from The Jackson Laboratory (Bar Harbor, ME, USA) and C57BL/6 mice ( $\mathrm{n}=12$, male) were purchased from Japan SLC (Shizuoka, Japan) and maintained in our laboratory. WBB6F $1-W / W^{v}$ mice ( $=3$, male) were purchased from Japan SLC. All experiments were done at the age of 8 to 12 weeks after birth. The use and treatment of the animals was in accordance with the Guidelines for Animal Experiments, the Regulations for Animal Research at University of Fukui. All efforts were made to minimize the number of animals used and their suffering.

\section{Ethical approval}

The animal experiments were approved by the Animal Research Committee, University of Fukui, and in accordance with the Regulations for Animal Research at University of Fukui.

\section{Immunohistochemistry}

For cryostat studies, the cecum was flushed with 0.01 $\mathrm{M}$ phosphate-buffered saline (PBS, $\mathrm{pH}$ 7.2) before being pinned to the Sylgard elastomer (Dow Corning Corporation, USA) floor of a dissecting dish. It was then fixed with fixative ( $2 \%$ paraformaldehyde made up in a $1.5 \%$ saturated picric acid solution, $0.1 \mathrm{M}$ phosphate buffer, $\mathrm{pH}$ 7.3) for $2 \mathrm{hr}$ at room temperature [7]. Following fixation, tissues were washed with PBS, immersed in $30 \%$ sucrose containing PBS, and embedded in Tissue-Tek (Miles, USA). Cryostat sections were cut at $10-\mu \mathrm{m}$ thickness using a Leica CM3050 cryostat and collected on poly-L-lysine-coated glass slides. Sections were washed with $0.3 \%$ Triton X-100 (PBST) for $2 \mathrm{hr}$ with several changes of the solution. After preincubation with normal donkey serum $(5 \%$ in PBS) for 1 $\mathrm{hr}$, sections were incubated with the following antibodies at room temperature: goat anti-c-Kit (AF1356, R\&D, 1:1000), rat anti-c-Kit (ACK4, CL8936AP, Cedarlane, 1:500), mouse anti- $\alpha$-smooth muscle actin-Cy3 (1A4, C6198, Sigma, $1: 3000)$, rat anti-smooth muscle myosin heavy chain (KM3669, 80058, Yamasa, 1:1000), rabbit anti-PGP9.5 (RA95101, UltraClone, 1:5000), rabbit anti-TMEM16A (ab53212, Abcam, 1:500), and goat anti-LRIG1 (AF3688, R\&D, 1:1000) [9, 12]. After sections were incubated overnight with primary antibodies, they were washed with PBS before incubation in the secondary antibody (Alexa Fluorcoupled donkey anti-IgG, Molecular Probes, 1:500) for $1 \mathrm{hr}$ at room temperature. After washing with PBS, specimens were nuclear stained with DAPI (Molecular Probes) and mounted with PermaFluor Aqueous Mounting Medium (Thermo Electron Corporation). The negative control consisted of normal goat, mouse, rabbit or rat sera used at the same concentration instead of the primary antibodies (Fig. 2).
Fluorescence was examined with a Leica TCS-SP2 confocal microscope with excitation wavelengths of 350 , 488 and $543 \mathrm{~nm}$. Images were collected and composed using Leica Confocal Software. Some confocal micrographs are the digital composites of several Z-series optical sections through the partial thickness of the musculature. Adobe Photoshop CS6 was used to compose the final images.

\section{Electron microscopy}

For conventional electron microscopy, tissues from two C57BL/6 mice were flushed with PBS before being pinned to the Sylgard dissecting dish and stretched to $80 \%$ of their resting length, and fixed with the fixative containing 3\% glutaraldehyde and 4\% paraformaldehyde in $0.2 \mathrm{M}$ phosphate buffer (PB, $\mathrm{pH} 7.4$ ) for $2 \mathrm{hr}$ at room temperature. After rinsing with $\mathrm{PB}$, the specimens were post-fixed with $1 \% \mathrm{OsO}_{4}$ in $\mathrm{PB}$ for $2 \mathrm{hr}$ at $4^{\circ} \mathrm{C}$. The specimens were then rinsed in distilled water, block-stained with $3 \%$ uranyl acetate solution, dehydrated in a graded series of ethyl alcohols and embedded in Epon resin (Epok 812, Oken, Japan). Ultrathin sections were cut using a Reichert ultramicrotome and double-stained with uranyl acetate and lead citrate for observation under Hitachi H-7650 transmission electron microscope.

For immunoelectron microscopy [10], tissues from two C57BL/6 mice were flushed with PBS before being pinned to a Sylgard dissecting dish and fixed with Zamboni's fixative plus $0.1 \%$ glutaraldehyde for $1 \mathrm{hr}$. The tissues were removed from the Sylgard dish and further immersed in Zamboni's fixative for $3 \mathrm{hr}$ at room temperature. A cryostat was then used to cut $10-\mu \mathrm{m}$ tissue sections, which were mounted on poly-L-lysine-coated slides. After washing with PBS, the sections were incubated for $1 \mathrm{hr}$ in normal goat serum $(10 \%$ in PBS), and then reacted with goat anti-c-Kit (1:3000 with PBS) for $24 \mathrm{hr}$ at $4^{\circ} \mathrm{C}$. After washing with PBS, sections were reacted with biotinylated horse anti-goat IgG (1:200 with PBS, Vector Laboratories, CA, USA) for $2 \mathrm{hr}$, and then reacted with avidin-biotinperoxidase complex (ABC Elite kit, Vector Laboratories) for $2 \mathrm{hr}$. The sections were incubated for several minutes with a solution containing $0.03 \%$ diaminobenzidine and $0.005 \% \mathrm{H}_{2} \mathrm{O}_{2}$ in $0.1 \mathrm{M}$ Tris- $\mathrm{HCl}(\mathrm{pH} 7.6)$. After coloration in diaminobenzidine solution, the specimens were postfixed using $1 \% \mathrm{OsO}_{4}$ in $0.1 \mathrm{M} \mathrm{PB}$ for $1 \mathrm{hr}$, block-stained with uranyl acetate, dehydrated in ethanol, and embedded in Epok 812. Ultrathin sections were examined using Hitachi H-7650 transmission electron microscope. The negative control consisted of normal goat serum used at the same concentration instead of the primary antibodies.

\section{Results}

We firstly examined ICC in the cecal musculature using c-Kit-copGFP reporter mouse (Fig. 1). ICC-MY that are distributed in the myenteric layer and ICC-IM in the 

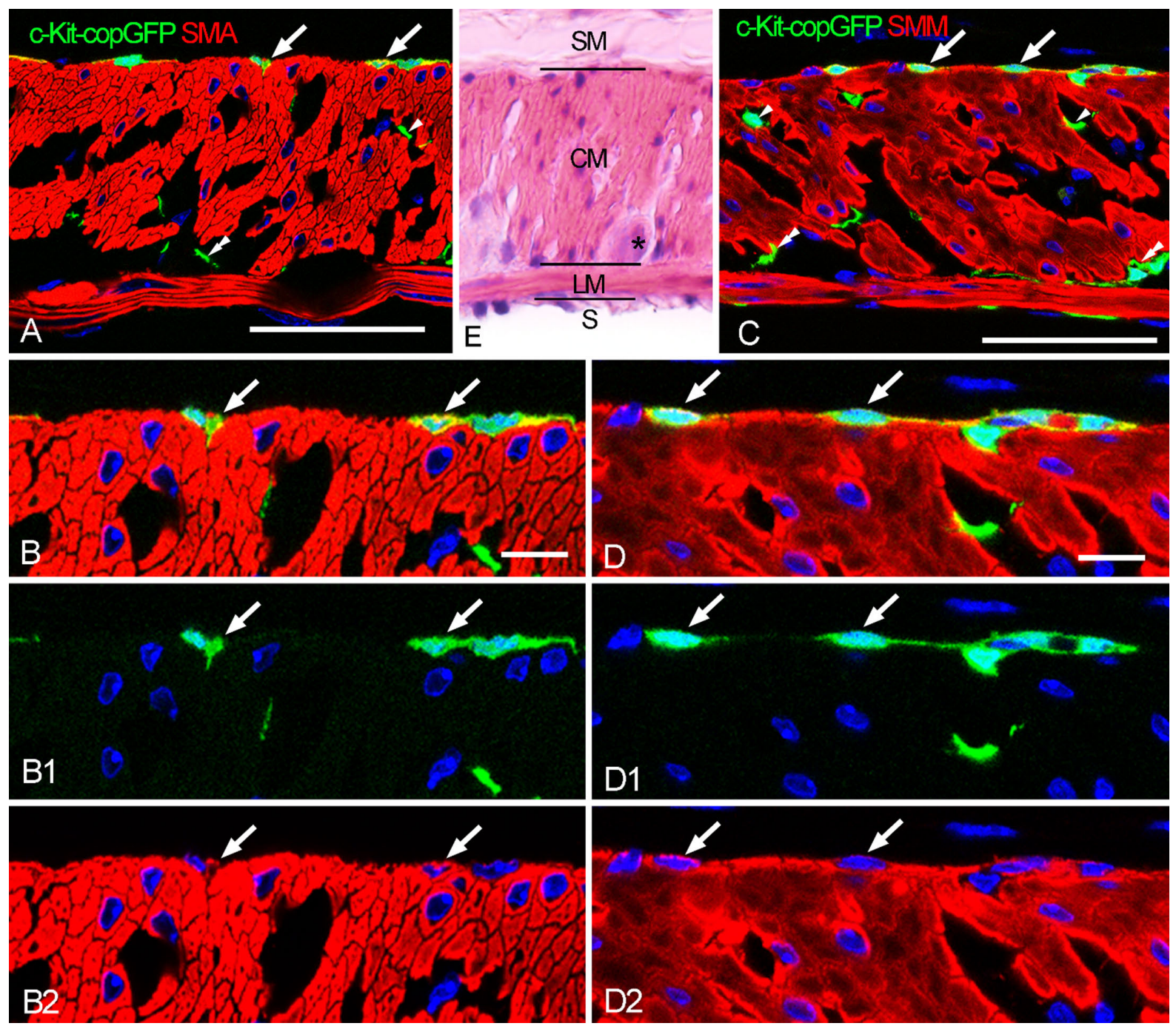

Fig. 1. Distribution and immunohistochemical properties of c-Kit-expressing cells in the c-Kit-copGFP mouse cecal musculature. $\alpha$-Smooth muscle actin (SMA) (red, A, B, B2) and smooth muscle myosin (SMM) (red, C, D, D2) immunoreactivities indicate smooth muscle cells that consist circular and longitudinal muscle layers. ICC-MY, ICC-IM and ICC-SS are negative for SMA and SMM immunoreactivities. At the submucosal surface of the circular muscle, c-Kit-copGFP-expressing cells (green) fall into line and show SMA and SMM immunoreactivities (arrows). Arrowheads show ICC-IM and double arrowheads show ICC-MY. B and D show enlarged view of A and C, respectively. B1 and B2, D1 and D2 show c-Kit-copGFP-expressing cells and smooth muscle cells, respectively. E shows C57BL/6 mouse cecum by hematoxylin-eosin staining to show submucosa (SM), circular muscle layer (CM), longitudinal muscle layer (LM) and serosal layer (S). Asterisk shows myenteric ganglion. Bars $=50 \mu \mathrm{m}(\mathbf{A}, \mathbf{C}), 10 \mu \mathrm{m}(\mathbf{B}, \mathbf{D})$.

circular and longitudinal muscle layers were clearly observed by GFP fluorescence as previously reported by cKit immunohistochemistry [9]. In the subserosal layer, ICC-SS were also observed same as in the colon [27]. Using smooth muscle-specific proteins such as $\alpha$-smooth muscle actin (SMA) and smooth muscle myosin (SMM), the musculature of murine cecum was clearly examined as circular and longitudinal layers. c-Kit-copGFP fluorescence was observed in myenteric layer, muscle layers, and subserosal layer as ICC-MY, ICC-IM, and ICC-SS, respectively.

c-Kit-copGFP fluorescence was also observed at the submucosal surface of the circular muscle (innermost layer of circular muscle), where ICC are not known to be dis- tributed. Because the immunoreactivity for c-Kit has not been reported in this area, except in our previous report [9], we carefully examined c-Kit immunoreactivity labeled by two antibodies, namely goat anti-c-Kit (AF1356, R\&D) and rat anti-c-Kit (CL8936AP, Cedarlane) combined with c-KitcopGFP fluorescence in the cecal musculature (Fig. 2A, B). Almost all c-Kit immunoreactivity was observed in c-KitcopGFP-expressing cells at the submucosal surface of the circular muscle layer as well as ICC in circular muscle, myenteric layer and serosal surface. c-Kit-expressing cells (c-Kit-copGFP cells or c-Kit-immunopositive cells) at the submucosal surface of the circular muscle layer (named as Kit-SM cells in this report) expressed smooth muscle marker proteins SMA and SMM, and showed slender cell 

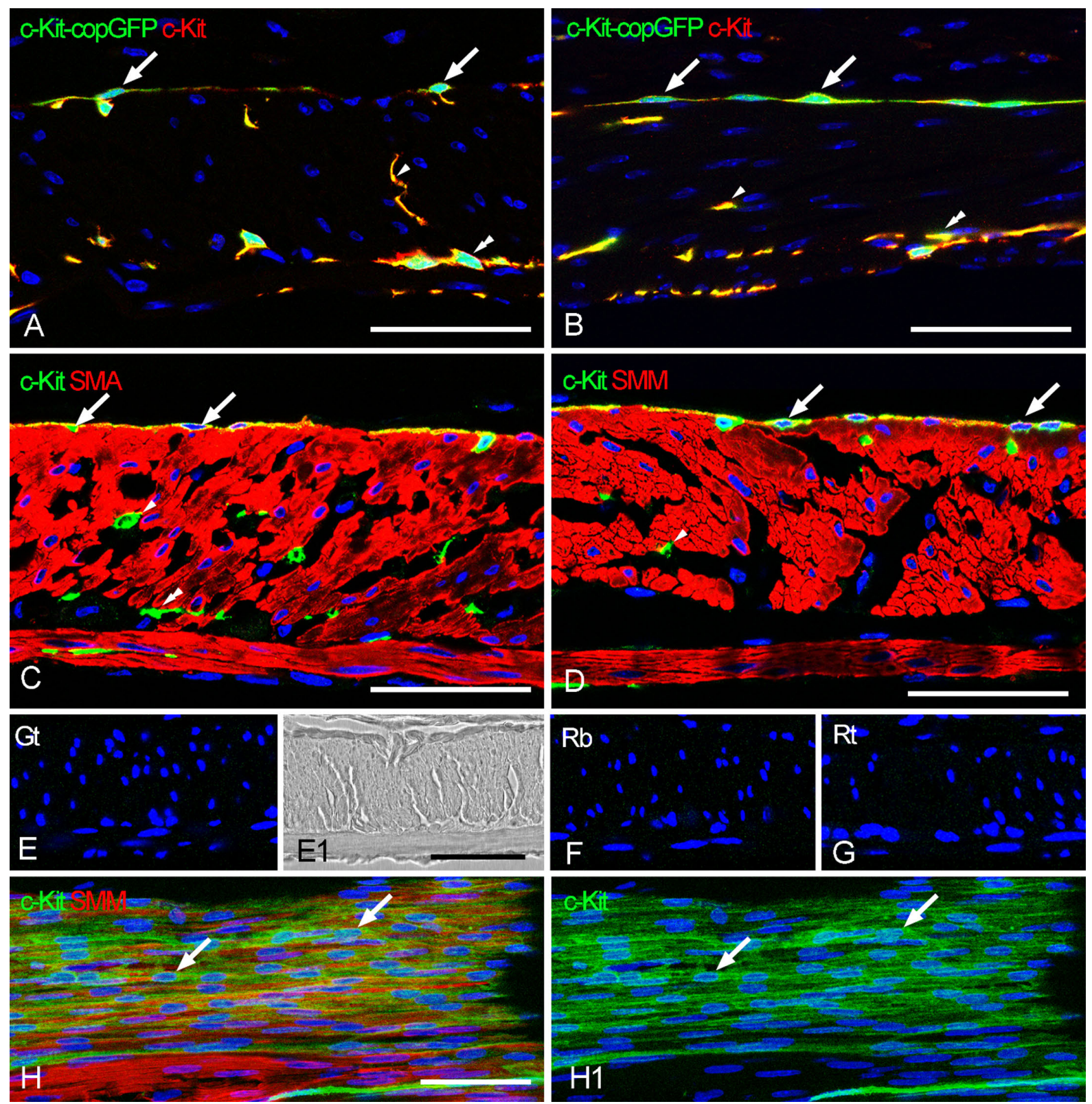

Fig. 2. c-Kit immunoreactivity along the submucosal surface of the circular muscle. A, B: Both c-Kit-copGFP fluorescence (green) and c-Kit immunoreactivity (red, A: rat antibody, B: goat antibody) distinctly label ICC-MY, ICC-IM and ICC-SS in musculature. Along the submucosal surface of the circular muscle, c-Kit-immunopositive cells also show c-Kit-copGFP fluorescence (arrows). A and B show longitudinal and circular sections of cecal musculature, respectively. c-Kit-immunopositive cells at the submucosal surface of the circular muscle have circumferentially long thin processes. C, D: c-Kit-immunopositive cells (green, arrows) along the submucosal surface of the circular muscle show SMA and SMM immunoreactivities (red). Arrowheads show ICC-IM and double arrowheads show ICC-MY. E-G show negative control figures by normal goat (E, Gt), rabbit (F, Rb) and rat (G, Rt) sera and Alexa Fluor 488 secondary antibodies. E1 shows transmission image of $\mathbf{E}$. H, H1: In the tangential sections of the circular muscle layer, cKit-immunopositive cells (green, arrows) line the submucosal surface of the circular muscle and are labeled by SMM (red). A, B: c-Kit-copGFP mice, C-H: C57BL $/ 6$ mice. Bars $=50 \mu \mathrm{m}$.

bodies with long cellular processes (Figs. 1 and 2). Under tangential sections of the circular muscle, c-Kitimmunopositive cells were situated at the surface of circular layer and observed as slender bipolar shape running parallel with smooth muscle cells (Fig. 2). In the submucosal surface of circular muscle, enteric nerve fibers labeled by PGP9.5 antibody were rare, and Kit-SM cells were not associated with enteric nerve fibers (Fig. 3).

Recent studies have reported multiple new molecules that are expressed in ICC. Of these, we detected TMEM16A immunoreactivity in both ICC and Kit-SM cells in murine cecum (Fig. 3). In addition, LRIG1 (leucine-rich repeats and immunoglobulin-like domains 1), which is a pan-ErbB negative regulator, was detected in 

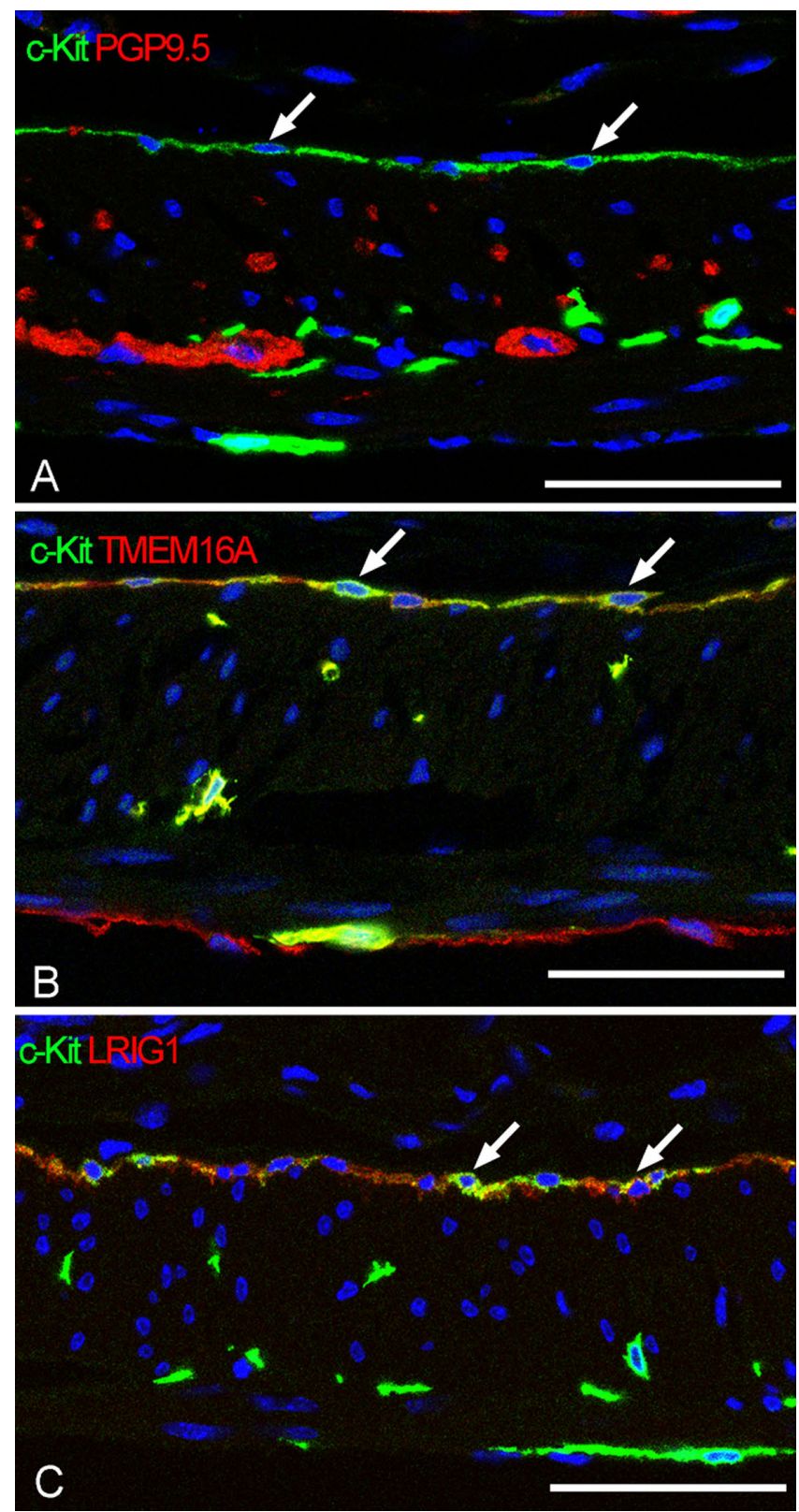

Fig. 3. Immunochemical properties of c-Kit-expressing cells at the submucosal surface of the C57BL/6 mouse cecum. A: PGP9.5immunopositive enteric nerves (red) in musculature are observed in myenteric layer and circular muscle layer. At the submucosal surface of the circular muscle, c-Kit-immunopositive cells (green, arrows) are not associated with enteric nerves. B: c-Kit-immunopositive cells (green, arrows) at the submucosal surface of the circular muscle and ICC show TMEM16A (red). Serosal mesothelial cells also express TMEM16A immunoreactivity. C: c-Kit-immunopositive cells (green, arrows) and negative cells at the submucosal surface of the circular muscle show LRIG1 immunoreactivity (red). Bars $=50 \mu \mathrm{m}$.

ICC-DMP in small intestine and ICC-SM in colon [14]. LRIG1 was also detected in Kit-SM cells as well as c-Kitimmunonegative cells at the submucosal surface of the circular muscle layer (Fig. 3).

ICC development is highly dependent on c-Kit signal-
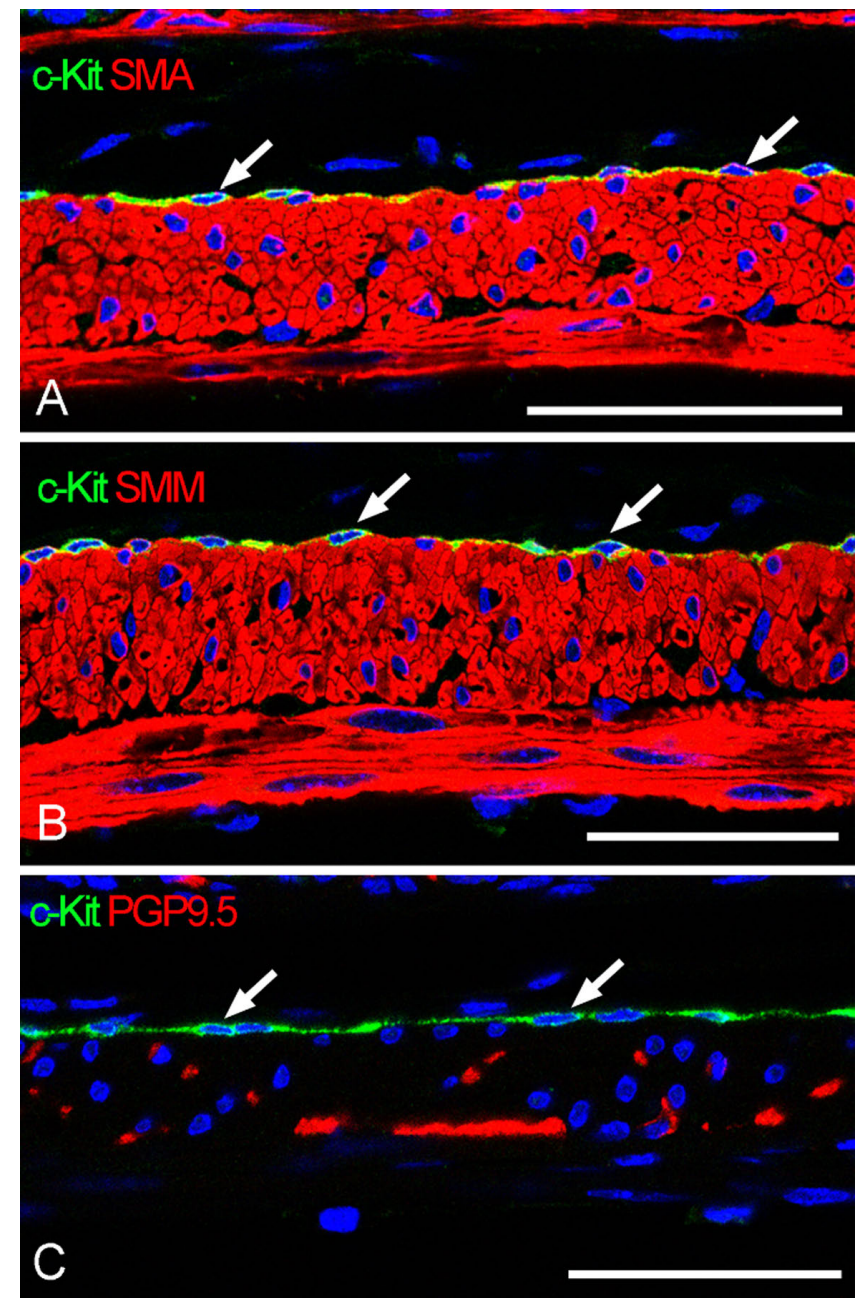

Fig. 4. Cecal musculature of $W / W^{v}$ mice. A, B: ICC-MY, ICC-IM, and ICC-SS are not observed in $W / W^{v}$ cecum. At the submucosal surface of the circular muscle, c-Kit-immunopositive cells (green, arrows) are observed with SMA (A) and SMM (B) immunoreactivities (red), same as wild type mice. C: PGP9.5-immunopositive nerves (red) are normally distributed in cecal musculature. c-Kit-immunopositive cells (green, arrows) at the submucosal surface of the circular muscle are not associated with enteric nerves. Bars $=50 \mu \mathrm{m}$.

ing and is inhibited in c-kit gene mutant mice ( $W$ mutant mice), which shows insufficient c-Kit signaling. In this study, we examined c-Kit immunoreactivites in $W / W^{v}$ mutant mice and confirmed ICC deficiency as reported previously [9] (Fig. 4). On the other hand, Kit-SM cells having c-Kit immunoreactivity were clearly observed at the submucosal surface of the circular muscle, and they expressed smooth muscle marker molecules SMA and SMM. The Kit-SM cells in $W / W^{v}$ mice were observed without association to enteric nerves, identical to normal mice.

Using electron microscope (Fig. 5), we examined the submucosal surface of the circular muscle and identified specialized smooth muscle cells that showed flattened cell bodies and long thin cellular processes. They contained thick and thin filaments and numerous caveolae and were 

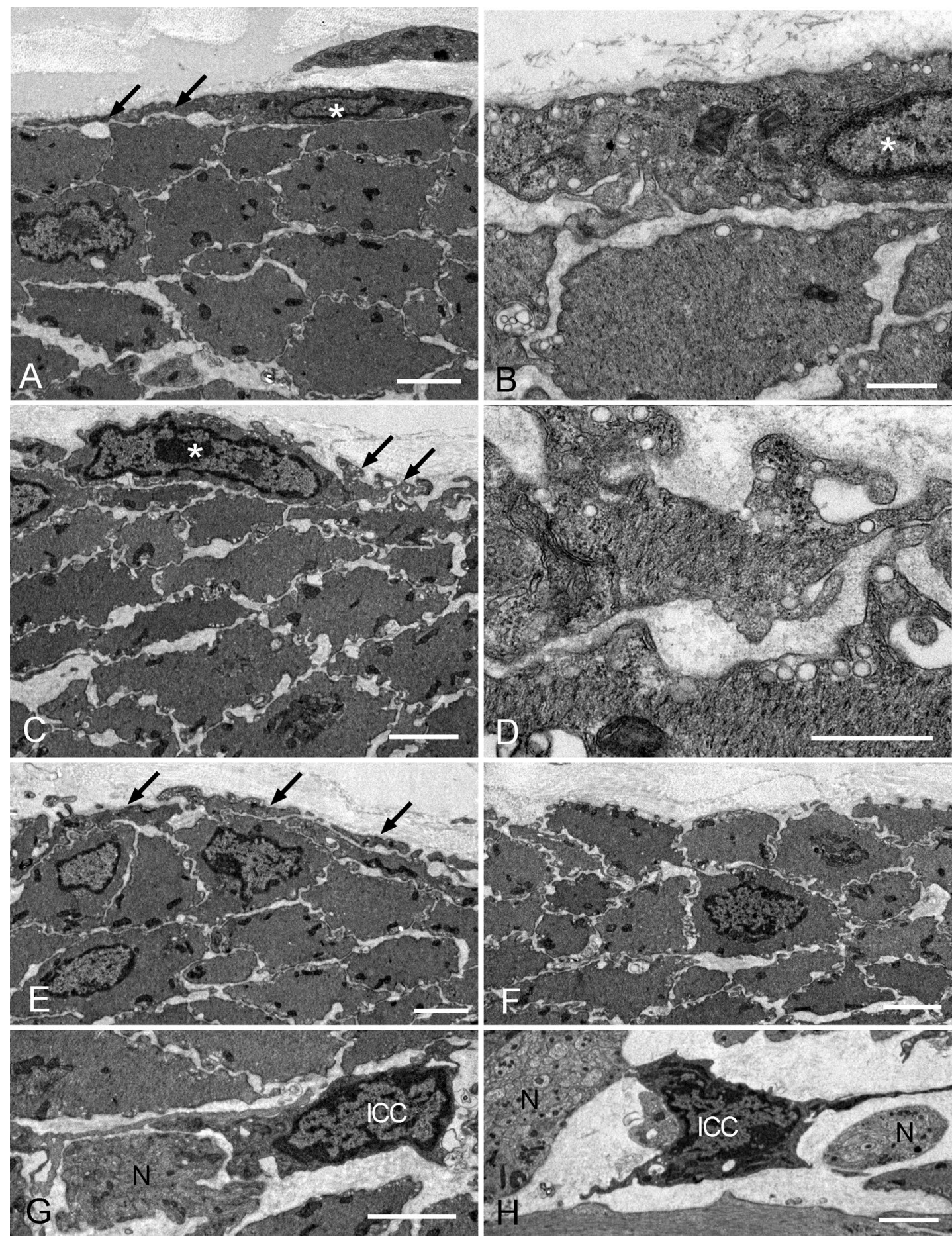

Fig. 5. Electron microscopic observations at the submucosal surface of the C57BL/6 mouse cecal musculature. In the innermost layer of circular muscle, there are elongated-shaped specialized smooth muscle cells (A, C, asterisks) that have long processes (arrows) along the submucosal surface of the circular muscle layer $(\mathbf{B}, \mathbf{E})$. These cells have thick and thin filaments and caveolae, and are surrounded by basal lamina (B, D). Several areas lack the long processes from specialized smooth muscle cells $(\mathbf{F})$. ICC-IM $(\mathbf{G})$ and ICC-MY $(\mathbf{H})$ in the musculature are observed near the enteric nerves $(\mathrm{N})$. Bars $=2 \mu \mathrm{m}(\mathbf{A}, \mathbf{C}, \mathbf{E}, \mathbf{F}, \mathbf{G}, \mathbf{H}), 0.5 \mu \mathrm{m}(\mathbf{B}, \mathbf{D})$.

surrounded by basal lamina. The specialized smooth muscle cells extended to the submucosal surface of the circular muscle layer. ICC in the cecal musculature were examined as observed previous studies [11, 19, 23]. Immunoelectron microscopic examination showed c-Kit-immunopositive cells located at the submucosal surface, and they had flattened cell bodies with thin elongated processes, as observed by conventional electron microscopy (Fig. 6). c-Kit- 

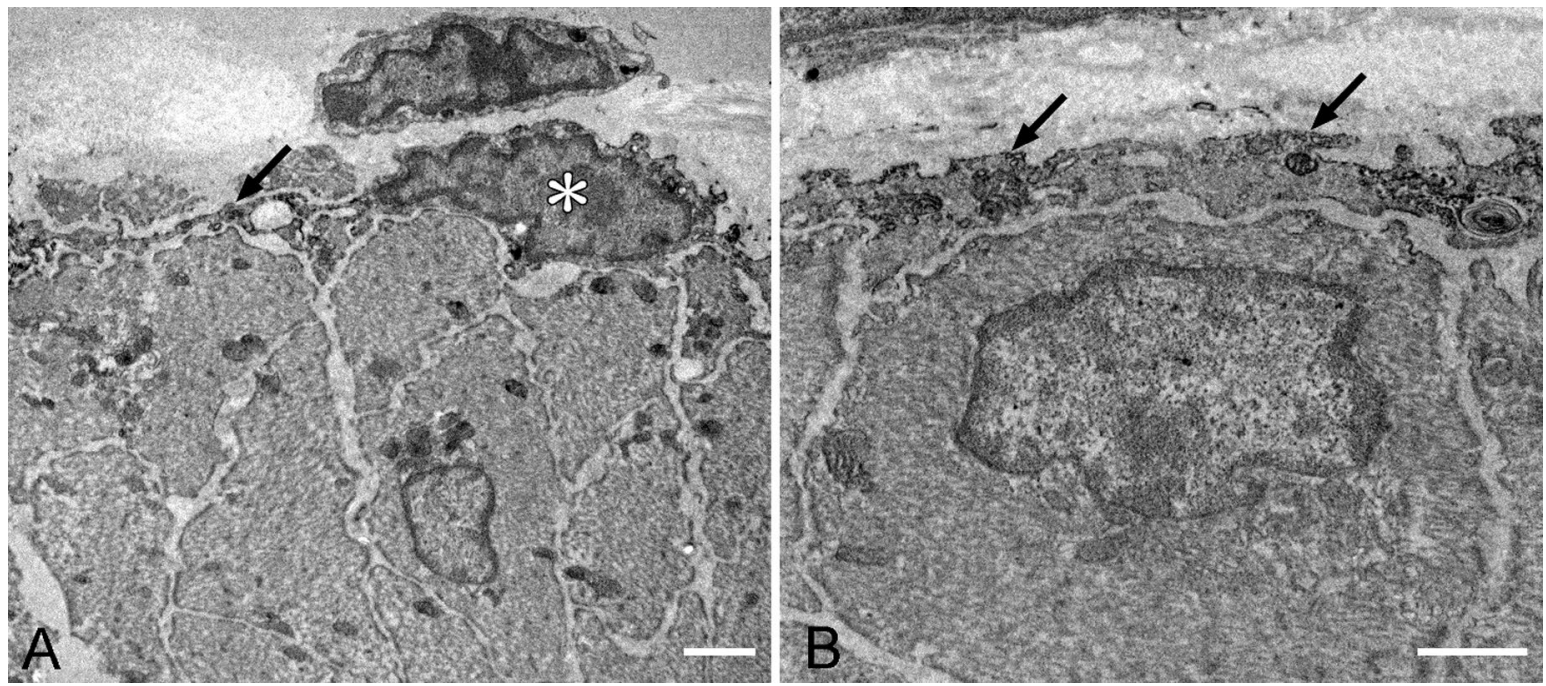

Fig. 6. c-Kit-immunopositive cells at the submucosal surface of the C57BL/6 mouse cecal musculature. Under electron microscope, c-Kit immunopositive-cells are located at the submucosal surface of circular muscle (A, asterisk) and show elongated cellular processes (arrows). Bars $=$ $1 \mu \mathrm{m}$

immunopositive cells corresponded to the specialized smooth muscle cells observed by conventional electron microscopy. The immunoelectron microscopic examination without c-Kit antibody did not show immunopositive staining.

\section{Discussion}

In this study, we clearly showed the smooth muscle cells that expressed c-Kit at the submucosal surface of the circular muscle (Kit-SM cells) in murine cecal musculature. These cells showed immunoreactivities toward smooth muscle actin and myosin, and thick and thin filaments at the ultrastructural level. These cells also showed ICC characteristics, with immunoreactivities for c-Kit, TMEM16A, and LRIG1. TMEM16A is a $\mathrm{Ca}^{2+}$-activated $\mathrm{Cl}^{-}$channel specifically expressed in ICC and regulates slow wave [4, 6]. LRIG1 is specifically expressed in ICC-DMP and ICCSM and regulates development of these cells in the postnatal period [14]. Taken together, we identified a new phenotype of smooth muscle cells at the submucosal surface of the circular muscle in murine cecum, which has not been reported earlier to the best of our knowledge.

The border between submucosa and tunica muscularis is known to be different among the sites of the GI tract. In the small intestine, the innermost layer of circular musculature is made of special smooth muscle cells called small and dark muscle cells [1,2]. In addition, between the innermost layer and outer layer of circular musculature, there are interstitial cells named ICC-DMP [23, 24]. These structures are completely defined by SMA/SMM-expressing smooth muscle cells and c-Kit-expressing ICC-DMP. In the colon, the submucosal surface of the circular muscle is defined with c-Kit-expressing cells known as ICC-SM [23, 24].
Both ICC-DMP and ICC-SM express c-Kit, TMEM16A, and LRIG1, but not SMA and SMM. Our study revealed the submucosal surface of cecum was composed of smooth muscle cells (Kit-SM cells) having ICC markers such as cKit, TMEM16A, and LRIG1.

Although the morphological aspects of tunica muscularis of guinea pig cecum have been investigated in detail [3], there are no reports regarding the characteristic smooth muscle cells at the submucosal surface of the circular muscle. In the guinea pig cecum, there is no specialized ICC arrangement at the submucosal surface of the circular muscle as well as ileocecal valve [19]. Our previous study of guinea pig tissues also indicated no specialized c-Kitimmunopositive cell arrangement at the submucosal surface of cecal musculature [11]. Ileocecal junction obtained from human specimens also showed no characteristic cell arrangement at the submucosal surface of the circular muscle [21]. To date, Kit-SM cells are specific in murine cecum; further critical study would be needed to realize these structures in different animals.

c-Kit expression in the GI musculature has been considered to be a marker of ICC. c-Kit regulates ICC-MY and ICC-IM development and c-kit gene mutations (loss of function mutations) lead to these cell deficiencies [25]. However, ICC-DMP in the small intestine and ICC-SM in the colon do not seem to be affected by c-kit gene mutations. In this study, the cecum of $c$-kit mutant $W / W^{v}$ did not show presence of ICC-MY and ICC-IM, although Kit-SM cells at the submucosal surface of the circular muscle were not affected by the $c$-kit gene mutations, as previously observed [9]. Kit-SM cells also expressed TMEM16A and LRIG1, which are known to be co-expressed in ICC-DMP and ICC-SM. These findings suggest that Kit-SM cells of cecal musculature are analogous to ICC, and have possibly 
pacemaker function similar to ICC-SM [23, 24]. The neuromuscular transmission achieved by ICC-DMP is not considered because of lack of nerve fibers around Kit-SM cells. On the other hand, Kit-SM cells were certainly smooth muscle cells, evident by immunohistochemical and electron microscopic studies. The function of Kit-SM cells as smooth muscle cells is not yet apparent, but these cells are considered to have specific function in the musculature.

In the developing intestinal musculature, c-Kitexpressing cells were observed in undifferentiated cells that differentiate into ICC in the embryonic period. On embryonic day 12.5 (E12.5), c-Kit is first detected in undifferentiated cells around the mucosa in the murine small intestine [28]. On E14.5, c-Kit-expressing cells begin to express SMM, and subsequently differentiate into c-Kit-positive/ SMM-negative cells (i.e. ICC) or c-Kit-negative/SMMpositive cell (i.e. smooth muscle cells) [13]. Based on these findings, undifferentiated c-Kit-expressing cells could differentiate to both ICC and smooth muscle cells. Gastrointestinal stromal tumors (GIST) that are c-Kit-expressing mesenchymal tumors in the GI tract are infrequently detected to have SMA immunoreactivity [18]. Thus, the cKit-expressing Kit-SM cells may potentially have proliferative or differentiating abilities.

TMEM16A is specifically expressed in ICC in GI tract and in smooth muscle cells in vasculature and airway [20]. Regarding the role in ICC, TMEM16A has been proposed to play a key role in the pacemaker function $[4,6]$ and possible role in the regulation of ICC proliferation [17]. TMEM16A is expressed in several cancers, including GIST, and is considered to regulate cancer growth and invasion [20]. The expression of TMEM16A in Kit-SM cells can be considered to suggest the proliferation potential, and not the pacemaker function in these cells.

LRIG1 is expressed specifically in ICC-DMP and ICC-SM in adult mice and intestinal smooth muscle cells in postnatal mice [14]. LRIG1 is distributed throughout the circular muscle layer in the postnatal period, and LRIG1and SMM-expressing cells differentiate to ICC (LRIG1and c-Kit-positive) and smooth muscle cells (LRIG1- and SMM-positive). Moreover, LRIG1 null mice were defective for ICC-DMP and ICC-SM; therefore, LRIG1 regulates the postnatal development of these ICC. In other tissues, LRIG1-expressing cells have been shown to be highly proliferative and contribute actively to the maintenance of the small intestine and colon [29], and changes in the expression of LRIG1 have been associated with malignant progression in several cancers [16]. Thus, LRIG1 may have roles in cell differentiation and proliferation. From the above, we suppose Kit-SM cells may also have potential to differentiate or proliferate in the musculature.

In summary, the present study has shown c-Kitexpressing smooth muscle cells (named Kit-SM cells) are situated at the submucosal surface of circular muscle layer. Kit-SM cells specifically expressed c-Kit, TMEM16A, LRIG1, smooth muscle actin, and myosin. Although the functional significance of Kit-SM cells has yet to be revealed, these cells can be considered to have proliferation or differentiation potential in the cecal musculature.

\section{Conflicts of Interest}

The authors declare that they have no conflicts of interest.

\section{Acknowledgments}

We would like to thank Editage (www.editage.com) for English language editing. This work was supported by JSPS KAKENHI Grant Numbers JP15K08150 (S.I.), JP17K10626 (K.H.), and JP17K09375 (S.H).

\section{References}

1. Gabella, G. (1972) Innervation of the intestinal muscular coat. $J$ Neurocytol. 1; 341-362.

2. Gabella, G. (1974) Special muscle cells and their innervation in the mammalian small intestine. Cell Tissue Res. 153; 63-77.

3. Gabella, G. (1981) On the musculature of the gastro-intestinal tract of the guinea-pig. Anat. Embryol. (Berl). 163; 135-156.

4. Gomez-Pinilla, P. J., Gibbons, S. J., Bardsley, M. R., Lorincz, A., Pozo, M. J., Pasricha, P. J., Van de Rijn, M., West, R. B., Sarr, M. G., Kendrick, M. L., Cima, R. R., Dozois, E. J., Larson, D. W., Ordog, T. and Farrugia, G. (2009) Ano1 is a selective marker of interstitial cells of Cajal in the human and mouse gastrointestinal tract. Am. J. Physiol. Gastrointest. Liver Physiol. 296; G13701381.

5. Huang, F., Rock, J. R., Harfe, B. D., Cheng, T., Huang, X., Jan, Y. N. and Jan, L. Y. (2009) Studies on expression and function of the TMEM16A calcium-activated chloride channel. Proc. Natl. Acad. Sci. U S A 106; 21413-21418.

6. Hwang, S. J., Blair, P. J. A., Britton, F. C., O’Driscoll, K. E., Hennig, G., Bayguinov, Y. R., Rock, J. R., Harfe, B. D., Sanders, K. M. and Ward, S. M. (2009) Expression of anoctamin 1/ TMEM16A by interstitial cells of Cajal is fundamental for slow wave activity in gastrointestinal muscles. J. Physiol. 587; 48874904.

7. Iino, S. (2000) Muscular innervation of the proximal duodenum of the guinea pig. Arch. Histol. Cytol. 63; 327-343.

8. Iino, S. and Horiguchi, K. (2006) Interstitial cells of Cajal are involved in neurotransmission in the gastrointestinal tract. Acta Histochem. Cytochem. 39; 145-153.

9. Iino, S., Horiguchi, S., Horiguchi, K. and Nojyo, Y. (2007) Interstitial cells of Cajal in the gastrointestinal musculature of $W$ mutant mice. Arch. Histol. Cytol. 70; 163-173.

10. Iino, S., Horiguchi, K. and Nojyo, Y. (2008) Interstitial cells of Cajal are innervated by nitrergic nerves and express nitric oxidesensitive guanylate cyclase in the guinea-pig gastrointestinal tract. Neuroscience 152; 437-448.

11. Iino, S., Horiguchi, K., Nojyo, Y., Ward, S. M. and Sanders, K. M. (2009) Interstitial cells of Cajal contain signalling molecules for transduction of nitrergic stimulation in guinea pig caecum. Neurogastroenterol. Motil. 21; 542-550, e512-543.

12. Izawa, Y., Kashii-Magaribuchi, K., Yoshida, K., Nosaka, M., Tsuji, N., Yamamoto, A., Kuroyanagi, K., Tono, K., Tanihata, M., Imanishi, M., Onishi, M., Sakiyama, M., Inoue, S. and Takahashi, R. (2018) Stem-like human breast cancer cells initiate vasculogenic mimicry on matrigel. Acta Histochem. Cytochem. 
$51 ; 173-183$.

13. Kluppel, M., Huizinga, J. D., Malysz, J. and Bernstein, A. (1998) Developmental origin and Kit-dependent development of the interstitial cells of Cajal in the mammalian small intestine. Dev. Dyn. 211; 60-71.

14. Kondo, J., Powell, A. E., Wang, Y., Musser, M. A., SouthardSmith, E. M., Franklin, J. L. and Coffey, R. J. (2015) LRIG1 regulates ontogeny of smooth muscle-derived subsets of interstitial cells of Cajal in mice. Gastroenterology 149; 407-419 e408.

15. Kunisawa, Y. and Komuro, T. (2008) Interstitial cells of Cajal associated with the submucosal plexus of the Guinea-pig stomach. Neurosci. Lett. 434; 273-276.

16. Lindquist, D., Kvarnbrink, S., Henriksson, R. and Hedman, H. (2014) LRIG and cancer prognosis. Acta Oncol. 53; 1135-1142.

17. Mazzone, A., Eisenman, S. T., Strege, P. R., Yao, Z., Ordog, T., Gibbons, S. J. and Farrugia, G. (2012) Inhibition of cell proliferation by a selective inhibitor of the $\mathrm{Ca}^{2+}$-activated $\mathrm{Cl}^{-}$ channel, Ano1. Biochem. Biophys. Res. Commun. 427; 248-253.

18. Mikami, T., Nemoto, Y., Numata, Y., Hana, K., Nakada, N., Ichinoe, M., Murakumo, Y. and Okayasu, I. (2013) Small gastrointestinal stromal tumor in the stomach: identification of precursor for clinical gastrointestinal stromal tumor using c-kit and alpha-smooth muscle actin expression. Hum. Pathol. 44; 2628-2635.

19. Miyamoto-Kikuta, S., Ezaki, T. and Komuro, T. (2009) Distribution and morphological characteristics of the interstitial cells of Cajal in the ileocaecal junction of the guinea-pig. Cell Tissue Res. 338; 29-35.

20. Oh, U. and Jung, J. (2016) Cellular functions of TMEM16/ anoctamin. Pflugers Arch. 468; 443-453.

21. Pollard, M. F., Thompson-Fawcett, M. W. and Stringer, M. D. (2012) The human ileocaecal junction: anatomical evidence of a sphincter. Surg. Radiol. Anat. 34; 21-29.

22. Ro, S., Park, C., Jin, J., Zheng, H., Blair, P. J., Redelman, D.,
Ward, S. M., Yan, W. and Sanders, K. M. (2010) A model to study the phenotypic changes of interstitial cells of Cajal in gastrointestinal diseases. Gastroenterology 138; 1068-1078 e1061-1062.

23. Rumessen, J. J. and Vanderwinden, J. M. (2003) Interstitial cells in the musculature of the gastrointestinal tract: Cajal and beyond. Int. Rev. Cytol. 229; 115-208.

24. Sanders, K. M., Koh, S. D. and Ward, S. M. (2006) Interstitial cells of Cajal as pacemakers in the gastrointestinal tract. Annu. Rev. Physiol. 68; 307-343.

25. Sanders, K. M. and Ward, S. M. (2007) Kit mutants and gastrointestinal physiology. J. Physiol. 578; 33-42.

26. Tamada, H. and Komuro, T. (2011) Three-dimensional demonstration of the interstitial cells of Cajal associated with the submucosal plexus in guinea-pig caecum. Cell Tissue Res. 344; $183-188$.

27. Tamada, H. and Kiyama, H. (2015) Existence of c-Kit negative cells with ultrastructural features of interstitial cells of Cajal in the subserosal layer of the $W / W^{v}$ mutant mouse colon. J. Smooth Muscle. Res. 51; 1-9.

28. Torihashi, S., Ward, S. M. and Sanders, K. M. (1997) Development of c-Kit-positive cells and the onset of electrical rhythmicity in murine small intestine. Gastroenterology 112; 144-155.

29. Wong, V. W., Stange, D. E., Page, M. E., Buczacki, S., Wabik, A., Itami, S., van de Wetering, M., Poulsom, R., Wright, N. A., Trotter, M. W., Watt, F. M., Winton, D. J., Clevers, H. and Jensen, K. B. (2012) Lrig1 controls intestinal stem-cell homeostasis by negative regulation of ErbB signalling. Nat. Cell Biol. 14; 401-408.

This is an open access article distributed under the Creative Commons Attribution License, which permits unrestricted use, distribution, and reproduction in any medium, provided the original work is properly cited. 